\title{
Endothelial Derived Micro Particles: Biomarkers for Heart Failure Diagnosis and Management
}

\author{
Alexander E. Berezin*
}

Chief of Cardiology Unit, Internal Medicine Department, State Medical University, Ukraine

Received: July 02, 2015; Accepted: July 31, 2015; Published: August 10, 2015

*Corresponding author: Alexander E. Berezin, Chief of Cardiology Unit, Internal Medicine Department, State Medical University, 26 Mayakovskyav, Zaporozhye, Ukraine, Tel: +380612894585; Fax: +380612894585; E-mail: dr_berezin@mail.ru

\begin{abstract}
Chronic Heart Failure (CHF) remains a leading cause of cardiovascular morbidity and mortality worldwide. The results from clinical trials on CHF have led to major improvements in diagnostic tools and in patient management. However, microvascular endothelial cell inflammation is considered a key stone of initial development of $\mathrm{CHF}$ and associates with vascular dysfunction and worse endothelial integrity. Endothelial-Derived Microparticles (EMPs) are probably novel biological markers of early stage endothelial injury and vascular tone disorders that might be detected prior to, clinically appeared CHF. Although measurement of EMPs is not still available for routine clinical use in many laboratories and requires specific technologies, there are large body of evidence that, elevated level of EMPs or imbalance between EMPs originated from activated and apoptotic endothelial cells may have predictive value for patients with CHF. The editorial comments are addressed to discuss about the predictive value of EMPs in CHF patients.
\end{abstract}

Keywords: Endothelial-derived Microparticles; Heart Failure; Endothelial Dysfunction; Biomarker; Cardiovascular Remodelling; Tissue Repair

Chronic Heart Failure (CHF) is a global medical problem that appears to be in close association with Cardiovascular (CV) morbidity and mortality [1]. Endothelial Dysfunction (ED) resulting in microvascular endothelial cell inflammation is considered a key stone of initial development of CHF. Theoretically, ED might detect at early stage of CHF and even prior cardiac dysfunction that opens new dawn for screening of the vulnerable patients using biomarkers reflected vascular injury and ED [2,3]. Recent studies have been shown that Endothelial-Derived Microparticles (EMPs) are probably novel biological markers of early stage endothelial injury and vascular tone disorders that might be detected prior to, clinically appeared CHF [3-6].

EMPs are heterogeneous population of vesicles (diameter 50-1000 $\mathrm{nm}$ ) that are released by cellular vesiculation and fission of the membrane of endothelial cells [7]. The triggers for the release of EMP into circulation are due to situational changes (physiological conditions, stress), micro environmental stimulation, coagulation / thrombosis, endotoxinemia, endothelial shear stress, growth factors, cytokines, ischemia and hypoxia [8]. Microparticles originated from activated and apoptotic derived endothelial cells play a pivotal role in intercellular information exchange through transfer of active molecules, microRNA, peptides, hormones, inflammatory factors and growth factors [9]. Elevated levels of apoptotic EMPs and low level of activated EMPs were frequently found in $\mathrm{CV}$ and metabolic diseases are closely associated with poor clinical outcomes [10]. Probably deficiency of proangiogenic EMPs and / or endothelial progenitor cells or coexisted elevated apoptotic derived EMPs might be considered as an impaired phenotype of circulating EMPs associated with poor prognosis, reflected a change in the apoptotic/reparative potential, being a putative indicator for vascular remodelling and endothelial dysfunction [11].

The difficulty of separating MPs from other types of cells, limits or efforts to extend actual cognitions in features affected biogenesis, secretion, and subsequent biological role of MPs. Current approach for measuring the MPs is based on the commonly used flow cytometry, Nanoparticle Tracking Analysis (NTA), Western blot analysis and electron microscopy. Unfortunately, all the above methods have crucial limitations regarding complicated assay and suffers from relatively low sensitivity and accuracy because of resolution problems occurring for the majority of commercially available flow cytometers [12]. Therefore, there are no standardized protocols regarding methods of isolation and analysis of MPs. Utilisation of flow cytometers specifically designed for analysis of MPs is probably to provide considerable methodological advantages and should be the preferable options [13]. In addition, problems with concentration limits of NTA measurements restrict the use of this method for clinical samples $[14,15]$. Western blot analysis and electron microscopy allows recognizing MPs depending on the determination of different markers and also represents a useful tool for examining particles. However, Western blot analysis and electron microscopy requires subsequent technical efforts and are much expensive [16]. Alternatively, recently recognized method for quantification and sizing of biological nanoparticlessurface plasmon resonance - based imaging microscopy (SPRi microscopy) might be significantly useful to resolve the majority of problems affected in MPs. As expected, simultaneous 
application of a high-sensitive fluorescent microscopy and SPRi microscopy should enhance the sensitivity and selectivity of a created biosensor platform $[17,18]$.

A Highly Sensitive Fluorescent (HSF) microscopy also permits to detect individual sub-micro and nano-MPs. As compared with SPRi microscopy, this technique could provide higher detection sensitivity due to large fluorescence excitation and a high effective quantum yield of fluorescence. Therefore, there are at least four methods that are not commercially available: Raman micro-spectroscopy, micro nuclear magnetic resonance technique, small-angle X-ray scattering and anomalous smallangle X-ray scattering $[19,20]$. All these methods are currently being explored to assay MPs, while an incorporation of these techniques into routine analytical practice is probably addressed in future.

Although elevated level of apoptotic EMPs have demonstrated high predictive value for $\mathrm{CHF}$ development and clinical outcomes [21-24], the role of activated EMPs is still not clear. Probably CHF development is the result of both disease-specific and traditional cardiovascular risk factors contributed an imbalance between apoptotic-derived EMPs and activated EMPs forming impaired phenotype. Whether impaired phenotype is useful to predict acute heart failure in patients with hypertension, cardiomyopathies, Diabetes Mellitus, stable coronary artery disease and asymptomatic atherosclerosis is not clear. It has been postulated that exiting apoptotic phenotype of EMPs may discuss a phenotypic marker coexisting in other cardiovascular risk factors such as dyslipidemia, obesity, diabetes, and hypertension. If this assumption is correct, EMP phenotyping is available for risk stratification among subjects in general population [25-28].

Future directions of clinical investigations regarding utility of EMPs as predictors of heart failure evolution, might open novel perspectives for personalization of the CHF care and probably incorporate serial measurements of EMPs in biomarker-guided therapy.

In conclusion, the contribution of EMPs in the pathogenesis of heart failure is close, impaired immune pattern of EMPs might be considered as a personalized marker of ED or vascular aging, while evidence of predictive value of this marker for CHF patients is limited.

\section{References}

1. Santulli G. Epidemiology of cardiovascular disease in the 21st century: updated numbers and updated facts. Journal of Cardiovascular Disease. 2013; 1(1): 1-2.

2. Tschöpe C, Van Linthout S. New Insights in (Inter) Cellular Mechanisms by Heart Failure with Preserved Ejection Fraction. Curr Heart Fail. Rep. 2014; 11(4): 436-44. doi: 10.1007/s11897-014-0219-3.

3. Berezin A, Zulli A, Kerrigan S, Petrovic D, Kruzliak P. Predictive role of circulating endothelial-derived microparticles in cardiovascular diseases. Clin Biochem. 2015; 48(9): 562-568. doi: 10.1016/j. clinbiochem.2015.02.003.

4. Nozaki T, Sugiyama S, Koga H, Sugamura K, Ohba K, Matsuzawa Y, et al Significance of a multiple biomarkers strategy including endothelial dysfunction to improve risk stratification for cardiovascular events in patients at high risk for coronary heart disease. J Am Coll Cardiol. 2009; 54(7): 601-8. doi: 10.1016/j.jacc.2009.05.022.

5. Montoro-García S, Shantsila E, Wrigley BJ, Tapp LD, Abellán Alemán J, Lip GY. Small-size Microparticles as Indicators of Acute Decompensated State in Ischemic Heart Failure. Rev Esp Cardiol (Engl Ed). 2015; doi: 10.1016/j.rec.2014.11.016.

6. Berezin AE, Kremzer AA, Samura TA, Martovitskaya YuV. Circulating Endothelial-Derived Apoptotic Microparticles in the Patients with Ischemic Symptomatic Chronic Heart Failure: Relevance of ProInflammatory Activation and Outcomes. Int Cardiovasc Res J. 2014; 8(3): 116-23.

7. Mause SF, Weber C. Microparticles: protagonists of a novel communication network for intercellular information exchange. Circ Res. 2010; 107: 1047-57. doi: 10.1161/CIRCRESAHA.110.226456.

8. Arraud N, Linares R, Tan S, Gounou C, Pasquet JM, Mornet S, et al. Extracellular vesicles from blood plasma: determination of their morphology, size, phenotype and concentration. J Thromb Haemost. 2014; 12(5): 614-27. doi: 10.1111/jth.12554.

9. Markiewicz M, Richard E, Marks N, Ludwicka-Bradley A. Impact of endothelial microparticles on coagulation, inflammation, and angiogenesis in age-related vascular diseases. J Aging Res. 2013; doi: $10.1155 / 2013 / 734509$.

10. Pirro M, Schillaci G, Bagaglia F, Menecali C, Paltriccia R, Mannarino $\mathrm{MR}$, et al. Microparticles derived from endothelial progenitor cells in patients at different cardiovascular risk. Atherosclerosis. 2008; 197(2): 757-67.

11. Berezin AE, Kremzer AA. Impaired phenotype of circulating endothelial microparticles in chronic heart failure patients: Relevance to body mass index. Diabetes Metab Syndr. 2015. doi: 10.1016/j. dsx.2015.04.003.

12. Lynch SF, Ludlam CA. Plasma microparticles and vascular disorders. Br J Haematol. 2007; 137(1): 36-48.

13. Burnier L1, Fontana P, Kwak BR, Angelillo-Scherrer A. Cell-derived microparticles in haemostasis and vascular medicine. Thromb Haemost. 2009; 101(3): 439-51.

14. Inal JM, Kosgodage U, Azam S, Stratton D, Antwi-Baffour S, Lange S. Blood/plasma secretome and microvesicles. Biochim Biophys Acta. 2013; 1834(11): 2317-25. doi: 10.1016/j.bbapap.2013.04.005.

15. Choi DS, Kim DK, Kim YK, Gho YS. Proteomics, transcriptomics and lipidomics of exosomes and ectosomes. Proteomics. 2013; 13(10-11): 1554-71. doi: 10.1002/pmic.201200329.

16. Dinkla S, Brock R, Joosten I, Bosman GJ. Gateway to understanding microparticles: standardized isolation and identification of plasma membrane-derived vesicles. Nanomedicine (Lond). 2013; 8(10): 1657-68. doi: 10.2217/nnm.13.149.

17. Shantsila E, Montoro-García S, Gallego P, Lip GY. Circulating microparticles: challenges and perspectives of flow cytometric assessment. Thromb Haemost. 2014; 111(6): 1009-14. doi: 10.1160/ TH13-11-0937.

18. Shpacovitch V. Application of Surface Plasmon Resonance (SPR) for the Detection of Single Viruses and Single Biological Nano-objects. J Bacteriology and Parasitology. 2012; 3: e110. doi: 10.4172/21559597.1000e110.

19.Zybin A, Kuritsyn YA, Gurevich EL, Temchura VV, Überla K, Niemax $\mathrm{K}$. Real-time detection of single immobilized nanoparticles by surface plasmon resonance imaging. Plasmonics. 2010; 5(1): 31-35. 
20.van der Pol E, Coumans F, Varga Z, Krumrey M, Nieuwland R. Innovation in detection of microparticles and exosomes. J Thromb Haemost. 2013; 11 Suppl 1: 36-45. doi: 10.1111/jth.12254.

21. Wang CC, Tseng CC, Hsiao CC, Chang HC, Chang LT, Fang WF, et al Circulating endothelial-derived activated microparticle: a useful biomarker for predicting one-year mortality in patients with advanced non-small cell lung cancer. Biomed Res Int. 2014: 173401. doi: 10.1155/2014/173401.

22.Sinning JM, Losch J, Walenta K, Böhm M, Nickenig G, Werner N. Circulating CD31+/Annexin V+ microparticles correlate with cardiovascular outcomes. Eur Heart J. 2011; 32(16): 2034-41. doi: 10.1093/eurheartj/ehq478.

23. Bernal-Mizrachi L, Jy W, Jimenez JJ, Pastor J, Mauro LM, Horstman LL, et al. High levels of circulating endothelial microparticles in patients with acute coronary syndromes. Am Heart J. 2003; 145(6): 962-70.

24. Montoro-García S, Shantsila E, Tapp LD, López-Cuenca A, Romero AI, Hernández-Romero D, et al. Small-size circulating microparticles in acute coronary syndromes: relevance to fibrinolytic status, reparative markers and outcomes. Atherosclerosis. 2013; 227(2): 313-22. doi: 10.1016/j.atherosclerosis.2013.01.028.
25. Nozaki T, Sugiyama S, Sugamura K, Ohba K, Matsuzawa Y, Konishi M, et al. Prognostic value of endothelial microparticles in patients with heart failure. Eur J Heart Fail. 2010; 12(11): 1223-8. doi: 10.1093/ eurjhf/hfq145.

26. Berezin AE, Kremzer AA, Samura TA, Martovitskaya YV, Malinovskiy YV, Oleshko SV, et al. Predictive value of apoptotic microparticles to mononuclear progenitor cells ratio in advanced chronic heart failure patients. J Cardiol. 2015; 65(5): 403-11. doi: 10.1016/j. jjcc.2014.06.014.

27. Nascimbene A, Hernandez R, George JK, Parker A, Bergeron AL, Pradhan S, et al. Association between cell-derived microparticles and adverse events in patients with nonpulsatile left ventricular assist devices. J Heart Lung Transplant. 2014; 33(5): 470-7. doi: 10.1016/j. healun.2014.01.004.

28. Garcia S, Chirinos J, Jimenez J, Del Carpio Muñoz F, Canoniero M, Jy w, et al. Phenotypic assessment of endothelial microparticles in patients with heart failure and after heart transplantation: switch from cell activation to apoptosis. J Heart Lung Transplant. 2005; 24(12): 21849. 